\title{
Real-Time Evolution of the Indium Tin Oxide Film Properties and Structure During Annealing in Vacuum
}

\author{
A. Rogozin, N. Shevchenko, M. Vinnichenko ${ }^{1}$, F. Prokert, V. Cantelli, \\ A. Kolitsch and W. Möller \\ ${ }^{1}$ Physics Department, Kyiv National Taras Shevchenko University, Kyiv 01033, Ukraine
}

Indium tin oxide (ITO) is widely applied as a transparent conductive oxide coating. A standard and successful industrial route of production is its deposition by magnetron sputtering from a compound (oxide) target [1]. To increase cost efficiency, it would be preferable to sputter reactively from a metal target at sufficiently high partial pressure of oxygen. However, under this condition, a satisfactorily low resistivity of the films cannot readily be obtained, [2] so that a deposition on heated substrates or post-deposition annealing is necessary. So far, the annealing processes for reactively sputtered ITO $[3,4]$ have only been studied for metal-rich films, in contrast to comprehensive studies after magnetron sputtering from ceramic targets [5-10]. Moreover, mainly isothermal heat treatment is considered in the literature, although annealing using a temperature ramp is of more relevance for practical application. Several investigations report on real-time in situ monitoring of the ITO film resistivity and reflectivity $[9,10]$, which is used for an indirect characterization of the crystalline structure of the films. This approach requires simplifying assumptions on the linear dependence of the resistivity or reflectivity on the crystalline fraction, and the stability of the film roughness during annealing. Direct investigations of the influence of heat treatment on the ITO film structure are so far limited to post-annealing studies by X-ray diffraction (XRD) and scanning or transmission electron microscopy [3-5,7,8,11].

Annealing of ITO is known to be very efficient in increasing the carrier concentration. It can be quite reasonably explained by the FrankKöstlin model [12] which accounts for tin donor activation at elevated temperatures. However, this model is valid only for crystalline ITO. The amorphous-to-crystalline transition in ITO during annealing is often assumed as the reason for this activation, but the physics behind the experimental observation is not clear. In the present letter we report the results of a real-time in situ investigation of the film properties and the structure evolution during annealing in vacuum.
The films are produced by reactive pulsed middle frequency magnetron sputtering using the facility and procedure described in Ref. 13. The chamber was pumped to a base pressure of $4 \times 10^{-4}$ $\mathrm{Pa}$ before deposition. The deposition runs were carried out for $2.5 \mathrm{~min}$ at an Ar flow of $30 \mathrm{sccm}$ (partial pressure $1.2 \mathrm{~Pa}$ ) and $\mathrm{O}_{2}$ flow of $64 \mathrm{sccm}$ $(0.3 \mathrm{~Pa})$. The films are grown on $\mathrm{Si}(100)$ substrates $\left(24 \times 12 \times 0.3 \mathrm{~mm}^{3}\right)$ covered with $\mathrm{SiO}_{2}$ $(510 \mathrm{~nm})$, which were not heated externally during deposition. The average thickness of the deposited films is $130 \mathrm{~nm}$. The as-deposited films show no crystalline peaks in the XRD patterns and are considered as amorphous.

The post-deposition annealing of identical ITO samples was carried out at two different experimental setups, the ROssendorf Beam Line (ROBL) at the European Synchrotron Radiation Facility in Grenoble, [14] and the ITO deposition facility at Forschungszentrum Rossendorf [13]. In both cases the total pressure of the residual gas was below $6 \times 10^{-4} \mathrm{~Pa}$. During annealing the sample was placed on a boralectric heater (Tectra, Germany). The sample temperature was controlled by a standard K-type thermocouple. In contrast to previous investigations [3-10] on isothermal annealing, in present work the annealing temperature $T_{a}$ was gradually increased from 20 to $330^{\circ} \mathrm{C}$ at a constant rate of approximately $5 \mathrm{~K} / \mathrm{min}$. In both experimental setups, the resistivity of the ITO films was monitored in situ by the four point probe technique.

In order to study the evolution of the ITO film structure in real time, a UHV annealing chamber, equipped with an X-ray transparent beryllium dome, was mounted on a six-circle goniometer. The X-ray diffraction experiment was performed in Bragg-Brentano geometry within the range of scattering angles of $27-37^{\circ}$. The incident $\mathrm{X}$-ray beam was monochromatized to $8.048 \mathrm{keV}$ $(\lambda=0.154 \mathrm{~nm})$. A multi-channel position sensitive detector allowed a fast scan acquisition time of $100 \mathrm{~s}$. The XRD data were evaluated by the PeakFit software (Jandel Scientific). 
A spectroscopic rotating compensator ellipsometer (M-2000, J.A Wollam Inc, USA) was used at FZR for in situ monitoring of the evaluation of the film optical properties. The ellipsometer is installed at the deposition chamber which is equipped with fused silica windows at angle of light incidence of $70.7^{\circ}$ with respect to the surface normal. The spectra of conventional spectroscopic ellipsometry (SE) parameters $(\Psi, \Delta)[15]$ within the probing photon energy range of $1.2-3.3 \mathrm{eV}$ were regularly recorded at $6 \mathrm{~s}$ intervals. The data were acquired and evaluated by the $\mathrm{WVASE}^{\circledR}$ software. A multilayer model [ambience/roughness layer/bulk ITO layer $/ \mathrm{SiO}_{2} / \mathrm{Si}$ ] was fitted to the measured $(\Psi, \Delta)$ spectra in ten different time intervals. The effect of elevated temperature on the optical properties of $\mathrm{Si}$ is accounted for by calibration at ten different temperatures. The dielectric function of the surface roughness layer was modeled as a $50 / 50 \mathrm{vol} . \%$ mixture of the ITO layer and voids, applying the Bruggeman effective medium approximation (EMA). The dielectric function of bulk ITO was parameterized in the Drude-Lorentz approach [15], which allows estimation of the free electron density $N_{D r}$ at a given temperature [16].

After increasing the annealing temperature $T_{a}$ up to $250 \pm 10^{\circ} \mathrm{C}$, the XRD patterns show only a broad amorphous peak around $32^{\circ}$, indicating the absence of any structural changes below this temperature. The evolution of the XRD patterns with annealing temperature within the range 200$320^{\circ} \mathrm{C}$ is shown in Fig. 1 . At $T_{a}=250^{\circ} \mathrm{C}$, the (222) and (400) peaks of crystalline $\operatorname{In}_{2} \mathrm{O}_{3}$ phase start to appear. Their intensity increases strongly as $T_{a}$ increases by approximately $30^{\circ} \mathrm{C}$, pointing to a rapid crystallization of the ITO film. The film becomes completely crystalline at $T_{a} \geq 280^{\circ} \mathrm{C}$ with a stable structure above. The calculation of the texture coefficient by known equations [17] shows that the film exhibits a weak (222) fibre texture in this state. The size of coherently diffracting domains was estimated by the Scherrer formula to $65 \pm 4 \mathrm{~nm}$.

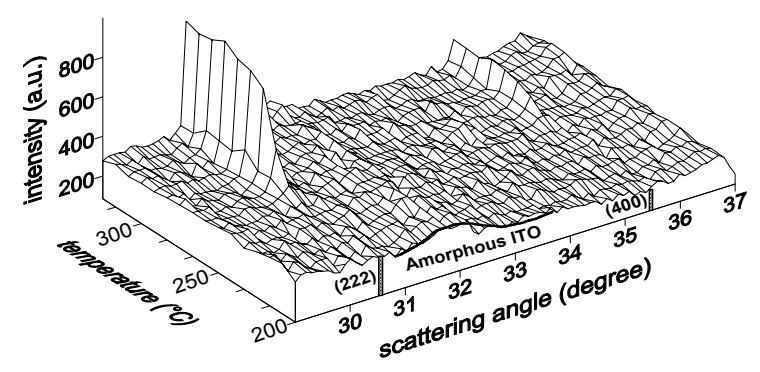

Fig. 1: Evolution of XRD patterns during annealing in vacuum.
The observed temperature of crystallization of $250^{\circ} \mathrm{C}$ is significantly larger than published values ranging from 150 to $200^{\circ} \mathrm{C}$, which are often cited $[9,10]$. However, the latter are deduced from post-deposition XRD studies of ITO layers produced on heated substrates [18]. During deposition, additional activation may occur due to plasmasurface interaction including fast ion bombardment, which might promote the crystallization at lower temperatures.

The temperature dependence of XRD integral intensity of the $\mathrm{In}_{2} \mathrm{O}_{3}(222)$ peak $\left(I_{(222)}\right)$ (Fig. 2(a)) has the typical S-like shape, being characteristic for the enhancement of the crystalline fraction during the amorphous-to-crystalline transition. The observed EMA roughness enhancement can be mainly related to the film crystallization because it occurs within the identical temperature range of $250-280^{\circ} \mathrm{C}$.

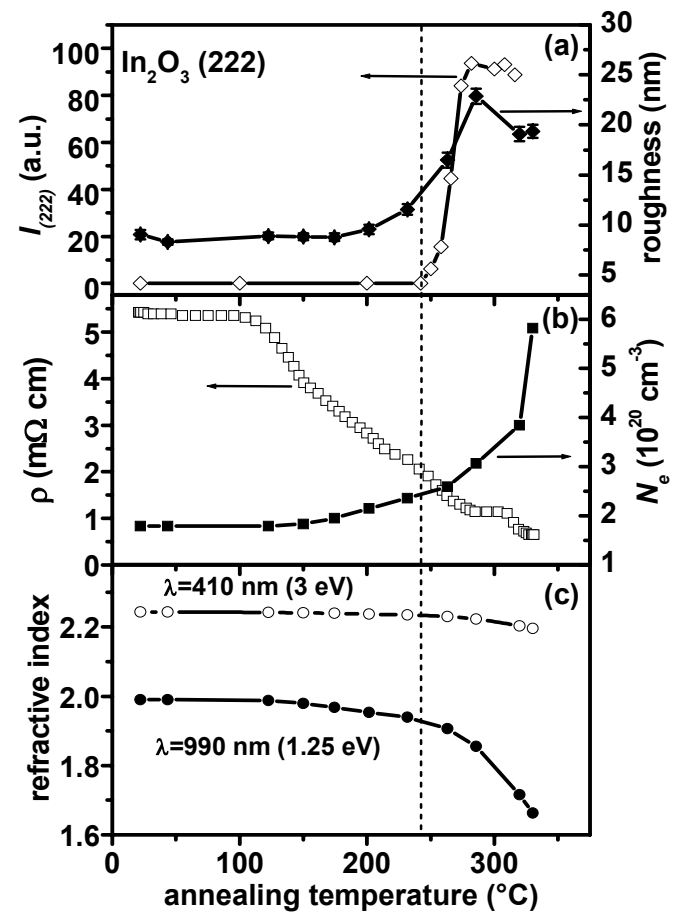

Fig. 2: The temperature dependence during annealing of the XRD integral intensity of the $\operatorname{In}_{2} \mathrm{O}_{3}(222)$ peak $\left(I_{(222)}\right)$ along with the EMA roughness (a), resistivity and free electron density $N_{D r}$ (b), and refractive index at two wavelengths (c).

Defining the degree of crystallization by $f=I_{(222)} / I_{C(222)}$, where $I_{C(22))}$ is the integral intensity measured at complete crystallization, its time dependence is analyzed by using the KolmogorovJohnson-Mehl-Avrami equation [19-21]

$$
f=1-\exp \left(-K t^{n}\right),
$$

where $n$ is a kinetic exponent and $K$ is a rate constant. A linear fit of the function $-\ln (1-f)$ 
plotted versus the acquisition time on a doublelogarithmic scale, yields $n=2.99 \pm 0.23$. Under the condition that the coherently diffracting domain size is smaller than the film thickness, this kinetic exponent indicates a three-dimensional crystallization process.

The observed temperature dependences of the resistivity in Fig. 2(b) and ellipsometry $(\Psi, \Delta)$ parameters (not shown here) indicate several stages with features at $T_{a}=110,150,280$ and $310^{\circ} \mathrm{C}$, in contrast to the more simple two-stage behavior of the resistivity and single-stage behavior of the reflectivity observed during isothermal annealing $[9,10]$. The temperature behavior of $N_{D r}$ in the Fig. 2(b) qualitatively agrees with a decrease of the resistivity, except in the temperature range of $280-310^{\circ} \mathrm{C}$.

The refractive index in Fig. 2(c) decreases at increasing annealing temperature above $150^{\circ} \mathrm{C}$. An even stronger decrease is observed above $T_{a}=250^{\circ} \mathrm{C}$ that relates to the free electron density behavior. The absolute values of the refractive index agree with the results reported elsewhere $[22,23]$. The decrease of the refractive index is ascribed to the enhancement of $N_{D r}$ [22].

From the stability of the film resistivity and refractive index it can be concluded that neither the free electron density nor the structure of the ITO films change during the first $20 \mathrm{~min}$ of annealing, corresponding to a temperature below $T_{a} \sim 120^{\circ} \mathrm{C}$. According to literature [11], the resistivity also does not change during isothermal annealing in vacuum at $T_{a}=125^{\circ} \mathrm{C}$ for the same time. At higher temperatures $\left(120-240^{\circ} \mathrm{C}\right)$ the free electron density rises in the amorphous ITO (Fig. 2(b)). According to Ref. 10, the increase of the free electron density can be attributed to the creation of oxygen vacancies due to relaxation of distorted In-O bonds in the amorphous phase. Since $\mathrm{Sn}$ is known not to be electrically active in amorphous ITO $[6,24]$ and does not affect the free electron density, any creation of substitutional Sn in the amorphous film can be neglected.

As the resistivity decreases faster than $N_{D r}$ increases, the free electron mobility can be assumed to be enhanced. However, due to the fit parameter correlation, it was not possible to extract from the SE data reliable values of the mobility in the whole temperature range, although the results suggest a mobility enhancement within the range $120-240^{\circ} \mathrm{C}$

The fast enhancement of the free electron density, $N_{D r}$, after the beginning of crystallization $\left(T_{a} \sim 250^{\circ} \mathrm{C}\right)$ can be explained by the onset of $\mathrm{Sn}$ donor activation $[9,10,24]$ in a growing crystalline phase. However, the SE is sensitive to the free electron density enhancement even in crystalline grains which are electrically insulated or have bad contact to each other [23]. These grains do not contribute to resistivity decrease until they are electrically connected. Possibly such connections are a reason for the drop of resistivity $\left(T_{a}>310^{\circ} \mathrm{C}\right)$ after a plateau observed at increasing $N_{D r}$ within the temperature range $280-310^{\circ} \mathrm{C}$.

The comparison of the graphs in Fig. 2 clearly shows that at constant heating rate the resistivity and optical properties depend nonlinearly on the crystalline fraction. This is in contrast to the linearity which is often assumed in the literature. A reason for this discrepancy might be the change of surface roughness in this particular case, which renders reliable monitoring of the film structure evolution by indirect techniques extremely difficult.

In summary, the structure and properties of ITO films deposited by reactive magnetron sputtering have been monitored in real time during annealing in vacuum, using three complementary in situ techniques. The direct observation of the structure yields a crystallization temperature of $250^{\circ} \mathrm{C}$. Even in the amorphous state the film resistivity decreases significantly at increasing temperature, probably due to relaxation of distorted In-O bonds in the amorphous phase, which leads to a free electron density enhancement by the creation of oxygen vacancies. The rapid crystallization is accompanied by film roughening and leads to a further decrease of the resistivity due to Sn donor activation.

The present results have been previously published as A. Rogozin et al.; Appl. Phys. Lett. 85 (2004) 212.

\section{References}

[1] B.G. Lewis, D.C. Paine, MRS Bull. 25 (2000) 22

[2] R.B.H. Tahar, T. Ban, Y. Ohya, Y. Takahashi, J. Appl. Phys. 83 (1998) 2631

[3] A.J. Steckl, G. Mohammed, J. Appl. Phys. 51 (1980) 3890

[4] S. Chaudhuri, J. Bhattacharyya, A.K. Pal, Thin Solid Films 148 (1987) 279

[5] Y. Shigesato, S. Takaki, T. Haranoh, J. Appl. Phys. 71 (1992) 3356

[6] Y. Shigesato, D.C. Paine, Appl. Phys. Lett. 62 (1993) 1268

[7] M. Higuchi, S. Uekusa, R. Nakano, K. Yokogawa, Jpn. J. Appl. Phys. 33 (1994) 302 
[8] S. Takayama, T. Sugawara, A. Tanaka, T. Himuro, J. Vac. Sci. Technol. A 21 (2003) 1352

[9] C.W. Ow-Yang, D. Spinner, Y. Shigesato, D.C. Paine, J. Appl. Phys. 83 (1998) 145

[10] D.C. Paine, T. Whitson, D. Janiac, R. Beresford, C. Ow Yang, B. Lewis, J. Appl. Phys. 85 (1999) 8445

[11] H. Morikawa, M. Fujita, Thin Solid Films 339 (1999) 309

[12] G. Frank, H. Köstlin, Appl. Phys. A: Solids Surf. 27 (1992) 197

[13] A.I. Rogozin, M.V. Vinnichenko, A. Kolitsch, W. Möller, J. Vac. Sci. Technol. A 22 (2004) 349

[14] W. Matz, N. Schell, G. Bernhard, J. Clausner, W. Oehme, F. Prokert, T. Reich, R. Schlenk, D. Pröhl, H. Funke, F. Eichhorn, M. Betzl, S. Dienel, V. Brendler, A. Denecke H. Krug, W. Neumann, G. Hüttig, P. Reichel, U. Strauch, J. Synchrotron Rad. 6 (1999) 1076
[15] B. Johs, J.A. Woollam, C.M. Herzinger, J. Hilfilker, R. Synowicki, C.L. Bungay, Crit. Rev. Opt. Sci. Technol. CR72 (1999) 29

[16] I. Hamberg, C.G. Granqvist, J. Appl. Phys. 60 (1986) R123

[17] H.-E. Cheng, M.-H. Hon, J. Appl. Phys. 79 (1996) 8047

[18] S. Muranaka, Y. Bando, T. Takada, Thin Solid Films 151 (1987) 355

[19] A. Kolmogorov, Izv. Akad. Nauk SSSR, Ser. Matem. 1 (1937) 355

[20] W. Johnson, R. Mehl, Trans. AIME 135 (1939) 416

[21] M. Avrami, J. Chem. Phys. 7 (1939) 1103

[22] B.N. De, Y. Zhao, P.G. Snyder, J.A. Woollam, T.J. Coutts, X. Li, Thin Solid Films 39, (1989) 647

[23] D. Mergel, Z. Qiao, J. Phys. D 35 (2002) 794

[24] J.R. Bellingham, W.A. Phillips, C.J. Adkins, J. Phys. Condens. Matter 2 (1990) 6207 